\title{
Simulations of surface muon production in graphite targets
}

\author{
Adriana Bungau, ${ }^{*}$ Robert Cywinski, and Cristian Bungau \\ University of Huddersfield, School of Applied Sciences, Huddersfield, HD1 3DH, United Kingdom \\ Philip King and James Lord \\ STFC-Rutherford Laboratory, Muon Facility, Didcot, OX11 OQX, United Kingdom \\ (Received 19 September 2011; published 22 January 2013)
}

\begin{abstract}
In this paper we present the results of GEANT4 simulations of the production of surface muons as a function of energy of the incident protons on a graphite target. A validation of the GEANT4 hadronic physics models has been performed by comparing the results with experimental data from the Lawrence Radiation Laboratory, United States. Considering the ISIS muon target as a reference, simulations have been performed to optimize the pion and muon production. Of particular significance, we predict that optimal surface muon production occurs at a relatively modest proton energy of $500 \mathrm{MeV}$. This will be of importance for the development of future $\mu \mathrm{SR}$ facilities.
\end{abstract}

DOI: 10.1103/PhysRevSTAB.16.014701

PACS numbers: $29.25 .-\mathrm{t}$

\section{INTRODUCTION}

Muon spin rotation, relaxation, and resonance, collectively known as $\mu \mathrm{SR}$, are uniquely sensitive probes of the distribution and dynamics of nuclear and atomic magnetic fields in materials of scientific and technological importance. Indeed $\mu$ SR has significantly improved our knowledge and understanding of the fundamental physical properties of superconductors, semiconductors, and magnetic systems [1]. The $\mu$ SR technique involves implanting positively charged polarized muons within a sample. The muons must be of sufficiently low energy to be stopped within a reasonable thickness (i.e. a few $\mathrm{mm}$ ) of sample where they couple to the local magnetic environment via their spin. The evolution with time of muon spin polarization within a sample is then detected via angular and temporal coordinates of the positrons emitted when the muons decay with a lifetime of $2.2 \mu$ s emitting a positron preferentially in the direction of the muons spin.

$\mu$ SR requires intense beams of positively charged spin polarized muons. Such beams are generally produced via the interaction of high energy proton beams with a light atomic mass (i.e. graphite) target and it is the decay (with a half-life of $29 \mathrm{~ns}$ ) of those positive pions created at rest at the surface of the target that leads to the emission of low energy or surface polarized positively charged muons with spins aligned antiparallel to their momentum. Typical proton-nucleon reactions producing pions at the production target are

\footnotetext{
*A.Bungau@hud.ac.uk

Published by the American Physical Society under the terms of the Creative Commons Attribution 3.0 License. Further distribution of this work must maintain attribution to the author(s) and the published article's title, journal citation, and DOI.
}

$$
\begin{array}{ll}
p+p \rightarrow p+n+\pi^{+} & p+n \rightarrow p+n+\pi^{0} \\
p+p \rightarrow p+p+\pi^{0} & p+n \rightarrow p+p+\pi^{-} \\
p+p \rightarrow d+\pi^{+} & p+n \rightarrow n+n+\pi^{+} .
\end{array}
$$

These are known as single pion production processes, and occur with an energy threshold of $280 \mathrm{MeV}$ in the laboratory frame. Above a laboratory frame energy threshold of $600 \mathrm{MeV}$, it is also possible to produce pairs of pions in the following proton-nucleon reactions:

$$
\begin{array}{ll}
p+p \rightarrow p+p+\pi^{+}+\pi^{-} & p+n \rightarrow p+n+\pi^{+}+\pi^{-} \\
p+p \rightarrow p+p+\pi^{0}+\pi^{0} & p+n \rightarrow p+n+\pi^{0}+\pi^{0} \\
p+p \rightarrow n+n+\pi^{+}+\pi^{+} & p+n \rightarrow n+n+\pi^{+}+\pi^{0} \\
p+p \rightarrow n+p+\pi^{+}+\pi^{0} & p+n \rightarrow d+\pi^{-}+\pi^{+} \\
p+p \rightarrow d+\pi^{+}+\pi^{0} & p+n \rightarrow d+\pi^{0}+\pi^{0} \\
& p+n \rightarrow p+p+\pi^{-}+\pi^{0} .
\end{array}
$$

In practice, appropriately intense beams of surface muons are produced by powerful high energy (500$1000 \mathrm{MeV}$ ) proton beams such as those in operation at large scale central facilities [2,3]: TRIUMF (Canada) and the Paul Scherrer Institute (Switzerland) produce continuous beams of muons for the international community of $\mu$ SR users, while ISIS (U.K.) and the newly commissioned J-PARC (Japan) provide an intense pulsed beam of muons. However, at each of these facilities there also exist other demands on the proton driver and consequently muon production rates may be suboptimal. At PSI, ISIS, and J-PARC the proton beams are optimized primarily for neutron beam production for neutron scattering studies of materials, while TRIUMF also serves the wider nuclear physics community.

The growing demand for muon beam time, and the greater scientific and technical capabilities afforded by 
even more intense muon beams, has led us to explore in detail the possibilities of further optimizing muon production rates. In this context we report here a series of GEANT4 simulations of proton-target interactions, and subsequent stationary positive pion and surface muon production, in which the simulations have been rigorously benchmarked against experimental data obtained at the Lawrence Radiation Laboratory. Our simulations may well prove useful both for increasing muon beam intensities at existing $\mu \mathrm{SR}$ facilities and for designing a new dedicated high intensity muon source for $\mu$ SR studies and fundamental physics [4].

\section{THE ISIS FACILITY}

Studies of target optimization for muon production were performed using the ISIS target geometry. Proton acceleration at ISIS begins with the ion source which produces negative hydrogen ions using an electric discharge. The negative ions are accelerated and separated into bunches by a radio frequency quadrupole accelerator which operates at $665 \mathrm{keV}, 202.5 \mathrm{MHz}$. Ion bunches are then further accelerated to $70 \mathrm{MeV}$ using a linear accelerator. Acceleration of the ions continues in the synchrotron, a $163 \mathrm{~m}$ circumference ring of magnets that bend and focus the beam. As the negative ions enter the synchrotron, a thin alumina foil strips away the electrons leaving a beam of protons. Once sufficient protons have been collected, they are further accelerated to $800 \mathrm{MeV}$. After almost 10000 revolutions the protons have separated out into two large bunches, the proton beam being double pulsed at $50 \mathrm{~Hz}$ with a nominal beam current of $200 \mu \mathrm{A}$. The proton bunches then travel on to collide with a tungsten

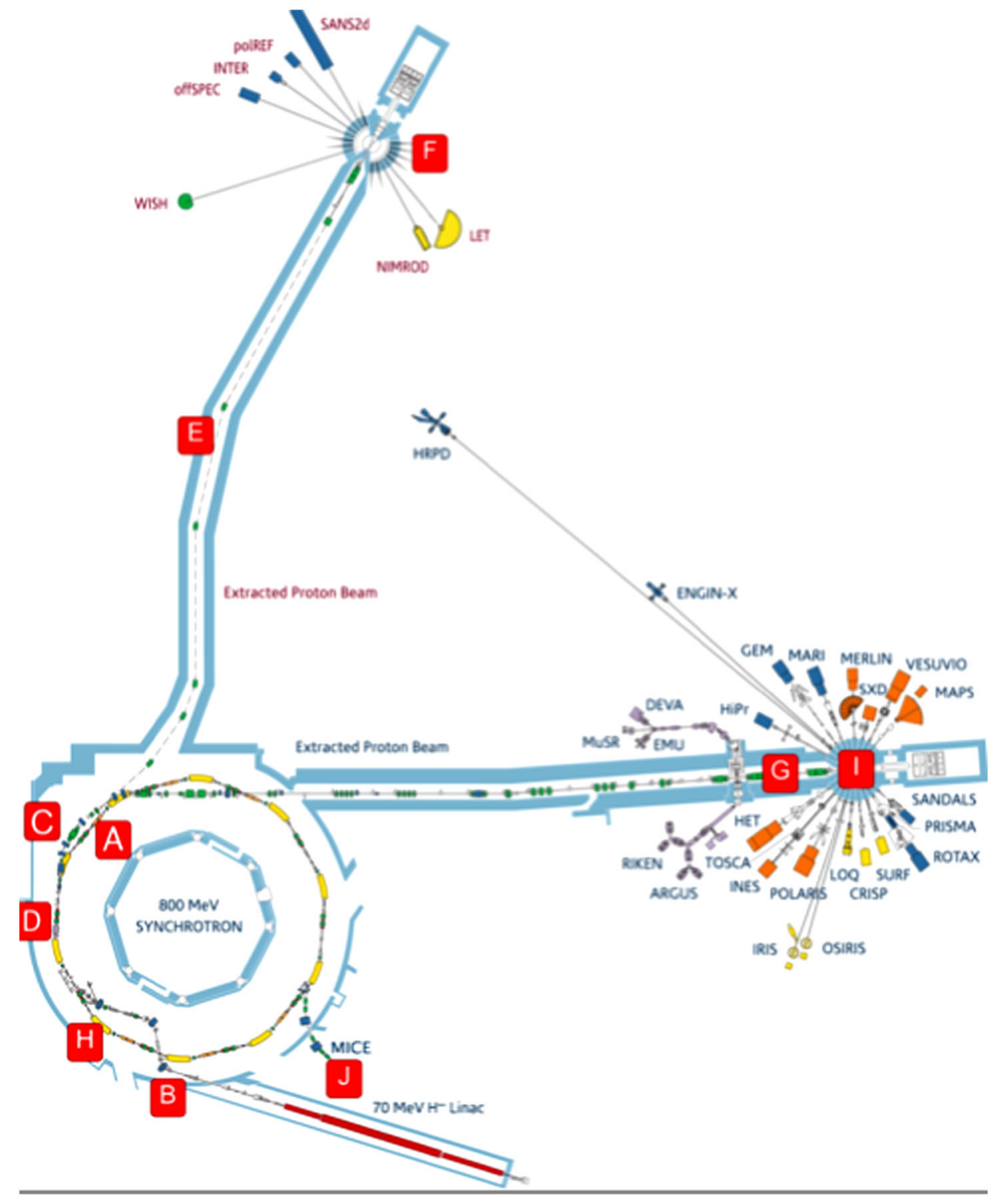

FIG. 1. Layout of the ISIS facility at the Rutherford Laboratory. The muon target (G) is situated $20 \mathrm{~m}$ upstream of the neutron target (I). The second neutron target is also shown (F). 
target to produce neutrons by spallation. A muon target is inserted into the proton beam line about $20 \mathrm{~m}$ upstream of the neutron target and pions are produced as a result of the proton interaction with the target nuclei. Pions produce muons which are directed to a suite of instruments optimized to explore different properties of materials (Fig. 1).

Although an ideal muon source does not exist as both continuous and pulsed sources have their advantages (frequency response for continuous sources and high data rates for pulsed sources), there are general requirements any muon source should have. The specifications of an ideal target are firstly a high yield of pions, and hence of muons resulting from the pion decay, and a small production of unwanted particles such as electrons and positrons, neutrons, scattered protons, and gamma rays. Low- $Z$ materials are used in order to maximize the pion production while minimizing multiple scattering of the proton beam itself. Moreover, the target should also generate little heat or dissipate heat easily, and have a low residual activity. The size of the proton beam at the target needs to be small, so that using electromagnetic optics, a small muon beam spot can be tailored to enable raster scanning of $\mu \mathrm{SR}$ samples, or the study of small single crystals.

The pulsed muon channel of the ISIS facility at Rutherford Appleton Laboratory has been successfully commissioned and operated for many years. The intermediate target used for muon production is an edge-cooled plate of graphite with dimensions $5 \times 5 \times 0.7 \mathrm{~cm}$ oriented at 45 degrees to the proton beam giving an effective length of $1 \mathrm{~cm}$ along the beam. The thickness of the intermediate target is limited by two factors: the proton transmission through the target must be kept at a reasonable level (usually 96\%) to prevent the loss in neutron intensity at the neutron facility and induced activity in downstream proton beam components produced by scatter in the muon target is required to be kept as low as possible (Fig. 2).

Water is used for cooling the target with the cooling system located outside the shielding of the proton beam.

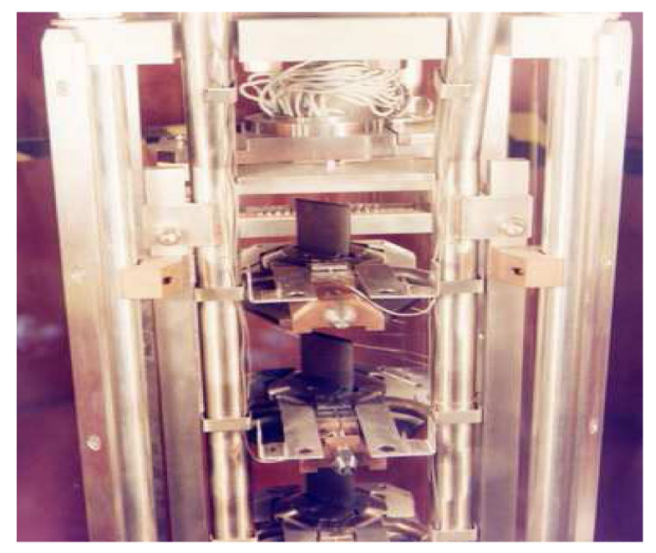

FIG. 2. Picture of the ISIS muon target. There are three targets on a rack which is lowered in the proton beam path during experiments.
The muon beam is extracted at 90 degrees to the proton beam. The muon beam is separated from the main proton beam by a thin aluminum window situated at $15 \mathrm{~cm}$ from the target center and having a diameter of $8 \mathrm{~cm}$. Experimentally, it was found that for $2.5 \times 10^{13}$ protons on target there are about 16000 positive surface muons counted at the entrance aperture of the beam window. The design and performance of the muon target is discussed in this paper together with the technical issues which must be addressed and particular attention is given to the relationship between primary proton beam energy and muon production as the first step towards any optimization.

\section{HADRONIC MODELS IN GEANT4}

Simulation studies of the muon target were performed using the Monte Carlo code GEANT4 [5] which is a toolkit for simulations of particle interactions in matter. A single hadronic model would not be able to support all user requirements; therefore GEANT4 provides a number of physics models, each model being defined for a given type of interaction within a specified range of energy. To cover all combinations of incident particle type, energy, and target material, different models are combined into a physics list in order to address the full spectrum of hadronic collisions. For this study, three such physics lists were considered: QGSP-BERT, QGSP-BIC, and QGSPINCL-ABLA. The physics list QGSP-BERT comprises the following physics models [6]: (i) Quark-Gluon String (QGS) model for all hadronic interactions above $12 \mathrm{GeV}$ followed by the Precompound model for preequilibrium and evaporation phases of the residual nucleus; (ii) LowEnergy Parametrized model for hadronic interactions between 9.5-25 GeV; (iii) Bertini Cascade (BERT) model which simulates the intranuclear cascade followed by preequilibrium and evaporation phases of the residual nucleus for proton, neutron, pion and kaon interactions with nuclei at energies below $9.9 \mathrm{GeV}$; (iv) parametrized models for all remaining hadrons; (v) parametrized capture and fission for low-energy neutrons; (vi) Chiral Invariant Phase Space (CHIPS) model of nuclear capture of negatively charged particles at rest; (vii) hadronic elastic scattering; (viii) quasielastic scattering; (ix) standard electromagnetic physics; (x) CHIPS model for gamma-nuclear and electronnuclear interactions; and (xi) parametrized muon-nuclear interactions.

The QGSP-BIC and QGSP-INCL-ABLA physics lists are similar except that the intranuclear cascades for protons, neutrons, and pions are modeled using the Binary Cascade Model and the INCL-ABLA model [7]. All three intranuclear cascade models applicable in the interest energy range for ISIS represent a theoretical approach to simulating hadronic interactions [8].

The Bertini Cascade Model [9] generates the final state for hadron inelastic scattering by simulating the intranuclear cascade. In this model, incident hadrons collide with 
protons and neutrons in the target nucleus and produce secondaries which in turn collide with other nucleons, the whole cascade being stopped when all the particles which can escape the nucleus have done so. Relativistic kinematics is applied throughout the cascade and the Pauli exclusion principle and conformity with the energy conservation law is checked. This model has been validated by extensive simulations on proton-induced reactions in various target materials and is validated up to $10 \mathrm{GeV}$ incident energy. This model is performing well for incident protons, neutrons, pions, photons, and nuclear isotopes.

In the Binary Cascade Model [10], the propagation through the nucleus of the incident hadron and the secondaries it produces is modeled by a cascade series of two-particle collision, hence the name binary cascade. Between collisions the hadrons are transported in the field of the nucleus by the Runge-Kutta method. This model reproduces detailed proton and neutron cross-section data in the region below $10 \mathrm{GeV}$, and pion cross-section data below $1.3 \mathrm{GeV}$.

To respond to the increasing user requirements from the nuclear physics community, the GEANT4 collaboration set a goal to complement the theory-driven models in this regime (the Bertini cascade and Binary Cascade being the most widely used) with the inclusion of the INCL code also known as Liege cascade, often used with the evaporation/

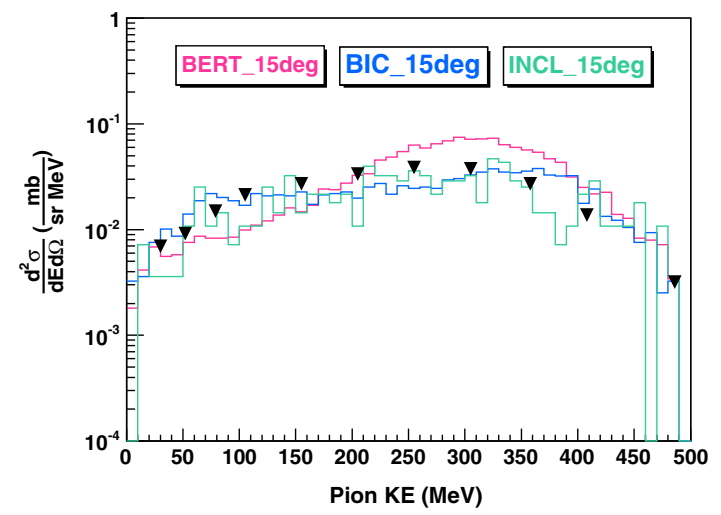

(a) Double differential cross section at 15 degrees

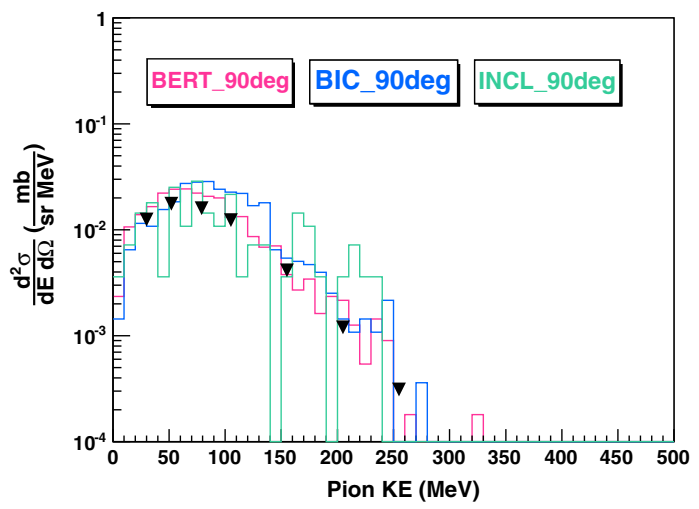

(c) Double differential cross section at 90 degrees fission code ABLA [11]. The model supports projectiles like protons, neutrons, pions, deuterium, tritium, helium, and alpha particles in the energy range $200 \mathrm{MeV}-3 \mathrm{GeV}$. The target material can be any element from carbon to uranium.

\section{GEANT4 MODEL VALIDATION}

Validation studies were being made by comparing results from thin target experiments with predictions from theoretical models of hadronic interactions. Thin target experimental data were used because they allow a clean and detailed study of single hadronic interactions.

Cochran et al. [12] performed experiments at the Lawrence Radiation Laboratory cyclotron which measured the pion production cross sections on targets over a wide range of production angles and pion energies. The experiment used the proton beam of the cyclotron, twelve different target materials, and a pion spectrometer consisting of a bending magnet and an array of 12 counter telescopes. The target materials used were $\mathrm{H}, \mathrm{D}, \mathrm{Be}, \mathrm{C}, \mathrm{Al}, \mathrm{Ti}, \mathrm{Cu}, \mathrm{Ag}, \mathrm{Ta}$, $\mathrm{Pb}$, Th, and liquid hydrogen. The beam passed through a premagnet collimator, a steering magnet, and a quadrupole doublet and then through a pipe in the shield, into the physics cave. The initial setup inside the physics cave was for forward angles. A quadrupole doublet was used

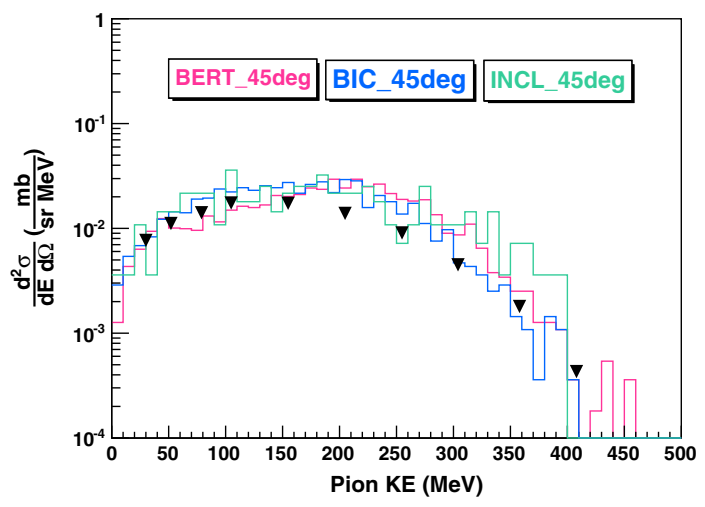

(b) Double differential cross section at 45 degrees

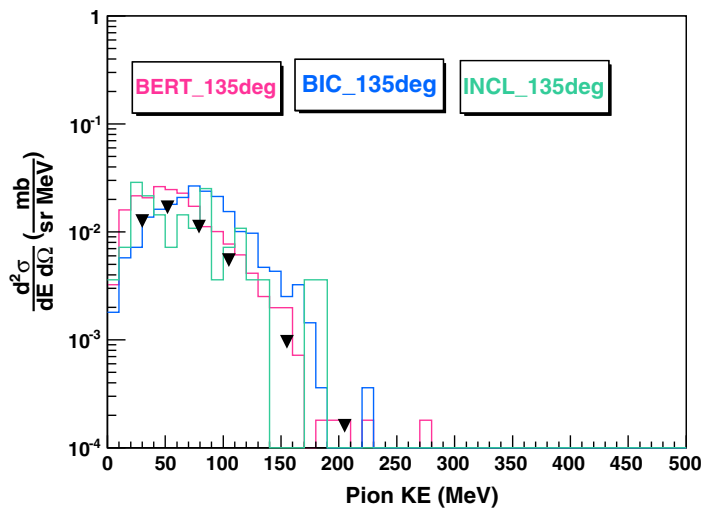

(d) Double differential cross section at 135 degrees

FIG. 3. Double differential cross section for positive pion production at 15, 45, 90, and 135 degrees with respect to the proton beam. Simulations using three physics lists (QGSP-BERT, QGSP-BIC, QGSP-INCL-ABLA) and experimental data are compared. 
to focus the beam to the primary target. The target was followed by a second doublet quadrupole used for stopping the beam in a steel block, $10 \mathrm{~m}$ downstream. When the apparatus was set up for backward angles, the second quadrupole doublet was used to focus the beam to a secondary target. After taking the backward-angle data, the setup was changed to forward angles with the premagnet collimator opened for these cross-section measurements. Several secondary beam channels over a wide range of angles were viewed by the magnetic spectrometer. The measured differential cross sections for pion production by $730 \mathrm{MeV}$ protons on targets provided a reliable guide for the design of pion beams at various meson facilities.

A thin $(1 \mathrm{~cm})$ carbon target was simulated with the GEANT4 code and four pion detectors were placed at 15 , 45,90 , and 135 degrees with respect to the proton beam. The solid angle from the target interaction point to each detector was $5 \mathrm{mrad}$. A beam of $10^{9}$ protons having an energy of $730 \mathrm{MeV}$ was sent to the carbon target. Three physics lists, QGSP-BERT, QGSP-BIC, and QGSP-INCLABLA, were used to model the proton interactions inside the target. The predictions of each model were then compared with the experimental data of $730 \mathrm{MeV}$ protons on a carbon target [13]. Figure 3 shows the positive pion production double differential cross sections for $15,45,90$, and 135 degrees. At small angles the Bertini model predictions underestimate the cross-section data for pion energies below $200 \mathrm{MeV}$ and overestimate the data above this value, while the Binary Cascade model gives a good overall description of data. At large angles the Binary model predictions overestimate the measured cross sections while the Bertini model predictions are more accurate. The predictions of the models are similar, therefore it is difficult to chose one model over another based on the rate at ISIS alone because the uncertainty in the solid angle being collected is larger than the rate differences. A comparison of the pion momentum spectra in various directions relative to the proton beam has been done by having in the simulation model eight detectors around the muon target and by comparing the results in the detectors that sit diagonally
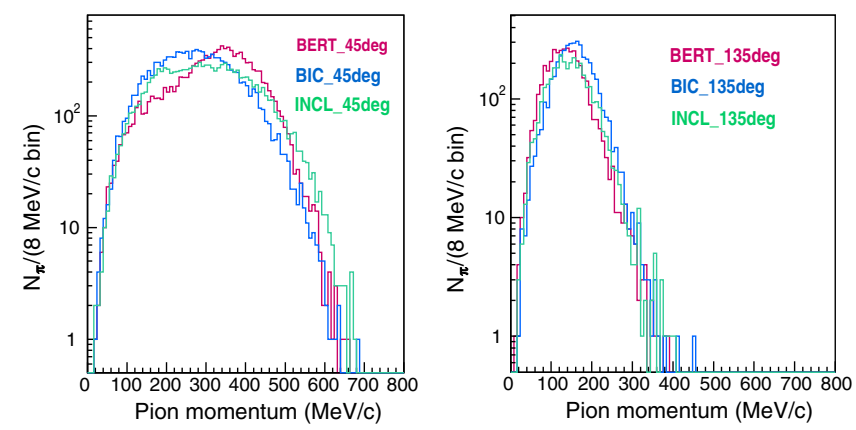

FIG. 4. Pion momentum spectra predicted by three hadronic models, Bertini, Binary, and INCL-ABLA. Detectors are placed diagonally opposed at 45 and 135 degrees with respect to the proton beam. opposed. Figure 4 shows the momentum spectra for pions forward scattered at 45 degrees and backscattered at 135 degrees relative to the proton beam and one can see that there are no significant differences between the models. Similar results have been seen for the other detectors.

These three models each have their strengths and weaknesses. It is therefore important to choose the most appropriate model for a particular application. The comparison of experimental and simulated pion production data, based upon each of these three models shown in Fig. 3, demonstrates that the general features of the data are reasonably well modeled by all three. However, the Binary Cascade and the INCL-ABLA models are both extremely CPU intensive [14]. Therefore, given the overall agreement between the three models we have elected to utilize the Bertini model for calculations.

\section{SIMULATIONS OF THE ENERGY DEPENDENCE OF MUON PRODUCTION RATES AT ISIS}

Typically muon production targets are used in transmission, with the transmitted proton beam being used for other particle production. For example at ISIS, only $4 \%$ of the proton beam is used for muon production with the remaining protons producing spallation neutrons. For simplicity we have used the ISIS geometry in the simulations; however, our results are quite general.

The muon production in a thin graphite target is an important topic given the widespread use of low-energy muons in various fields of physics. The current simulation studies have the scope to determine the optimal incident proton energy for pion and surface muon production and further work will address other aspects of target design like target material, different geometries, etc. For muon experiments it is desirable to optimize the number of muons produced while keeping in mind the limitations of the target geometry and the proton transmission. An optimization of the collection geometries would also be a bonus. The ISIS target geometry was modeled in the computer code, together with the muon beam window and two collimators placed after the muon target [15]. The purpose of the collimators is to stop any pions and neutrons formed at low angles, or protons scattered through larger than average angles, which would otherwise hit the beam pipe or quadrupole magnets between the muon and neutron targets. The collimators are $40 \mathrm{~m}$ long angled cones of $\mathrm{Cu}$. The first collimator has an inner radius of $37.5 \mathrm{~mm}$ and an outer radius of $54.15 \mathrm{~mm}$ and intercepts protons scattered beyond $41.6 \mathrm{mrad}$. The second collimator has an inner radius of $51.0 \mathrm{~mm}$ and an outer radius of $61.4 \mathrm{~mm}$ and intercepts protons at angles greater than $28.8 \mathrm{mrad}$. A proton beam having $10^{9}$ protons and an energy of $800 \mathrm{MeV}$ was sent to the target. In all simulations it is assumed that the proton beam have zero energy spread (the actual value at ISIS is $1 \mathrm{MeV}$ ). 


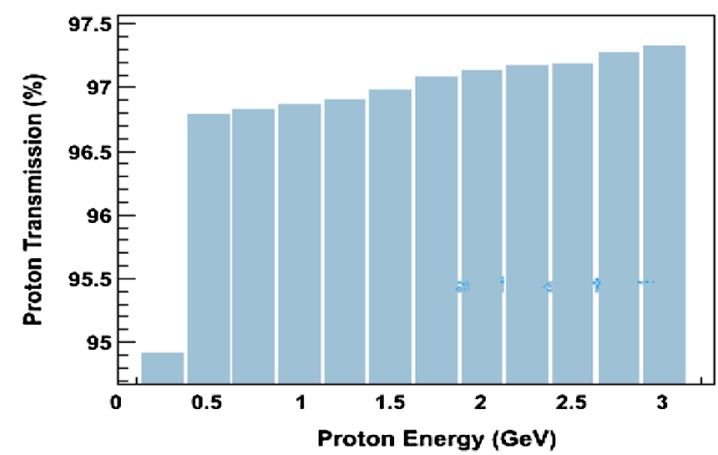

FIG. 5. Proton transmission through the muon target as a function of the proton beam energy.

\section{A. Proton transmission}

Because the muon facility at ISIS runs in parallel with the neutron facility, the proton transmission through the muon target defined as the fraction of protons passing through the collimation system, must be taken into account. If we increase the energy of the proton driver, the proton transmission through the muon target is increasing (Fig. 5).

As a consequence thicker targets could be used at higher energies (Fig. 6). However, for the current study the fixed target thickness was considered in all simulations.

\section{B. Pion production}

At ISIS, the muon beam has a vertical acceptance of $\pm 0.5 \mathrm{~cm}$ and a horizontal acceptance of $\pm 3 \mathrm{~cm}$. The acceptance angle is $35 \mathrm{mrad}$ in the horizontal direction and $180 \mathrm{mrad}$ in the vertical direction. Only positive decay muons having a momentum in the range $25.175-27.825 \mathrm{MeV} / c$ per unit charge are accepted by the muon beam line. For the purpose of the efficient pion production, the primary proton beam energy was typically chosen to be greater than twice the pion mass and simulations were then performed for different incident proton energies with the aim of optimizing the parameters of the proton beam. The pion yield increases rapidly with energy, as shown in Fig. 7.

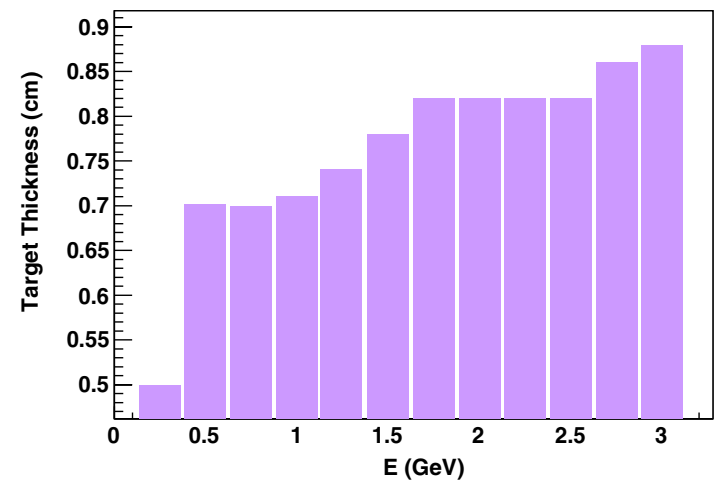

FIG. 6. Target thickness for $96 \%$ proton transmission through the muon target as a function of proton beam energy.

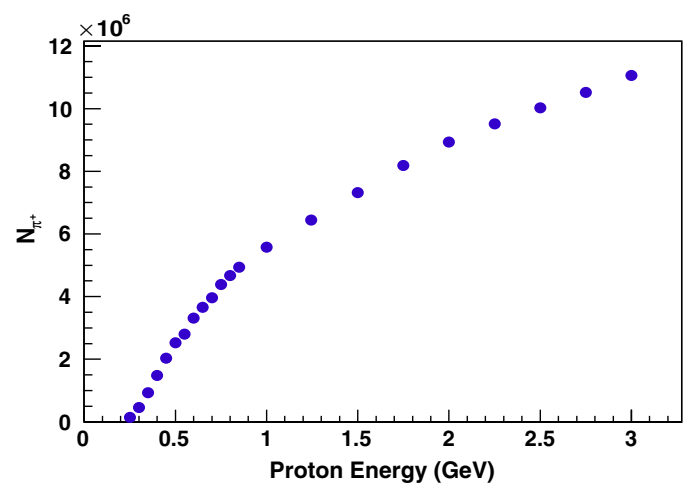

FIG. 7. Variation of pion yield with proton energy.

A fraction of the pions produced inside the target have low energy and stop at the target surface layer after having completely lost their momentum inside the target itself. They decay at rest producing monoenergetic surface muons with a high polarization. There is also another fraction of pions which decays in flight in the free space close to the production target and, because the momenta of the parent pion is unknown, the muons produced have a lower net polarization. The threshold of the single pion production reactions as a result of proton-nucleon interactions inside the target is typically $280 \mathrm{MeV}$ in the laboratory frame. To obtain a maximum number of single pions the incident proton beam should have an energy in the
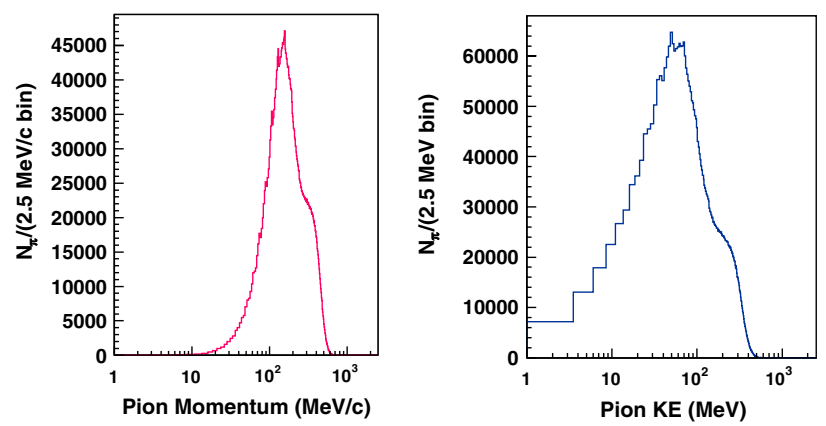

FIG. 8. Pion momentum and energy spectra for incident proton energy of $750 \mathrm{MeV}$.
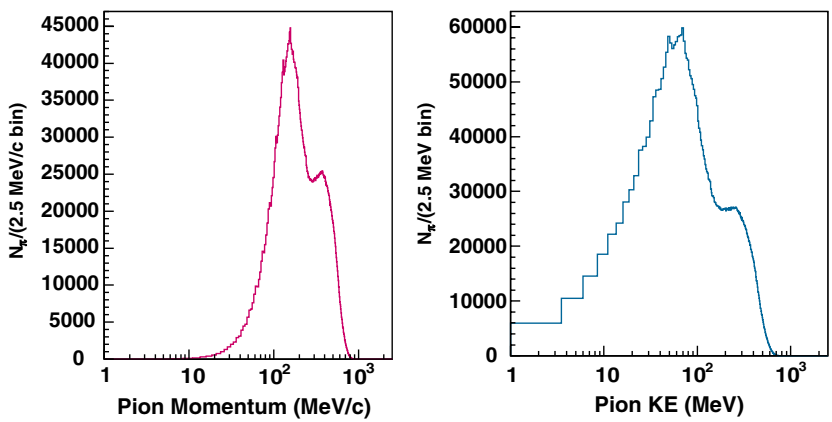

FIG. 9. Pion momentum and energy spectra for incident proton energy of $1 \mathrm{GeV}$. 


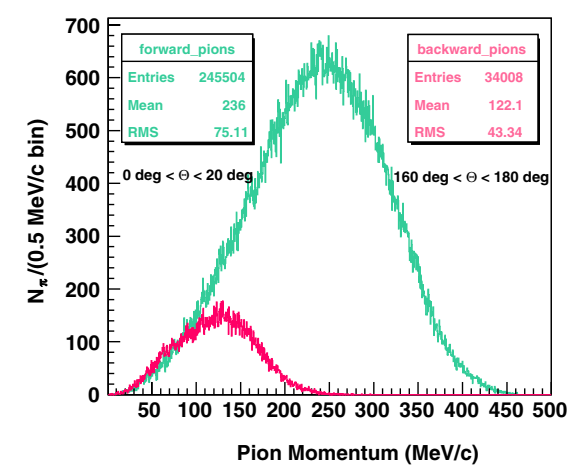

(a) $500 \mathrm{MeV}$ incident protons

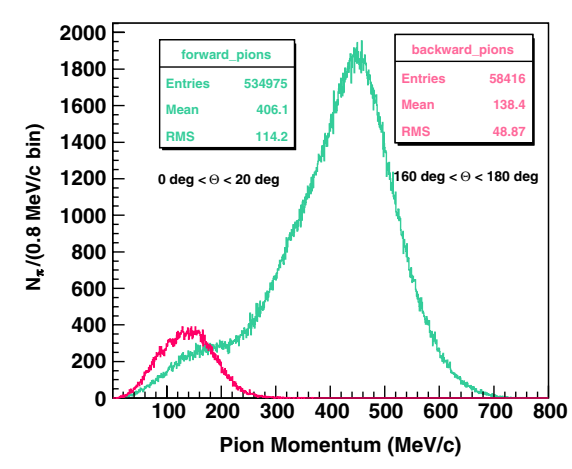

(b) $800 \mathrm{MeV}$ incident protons

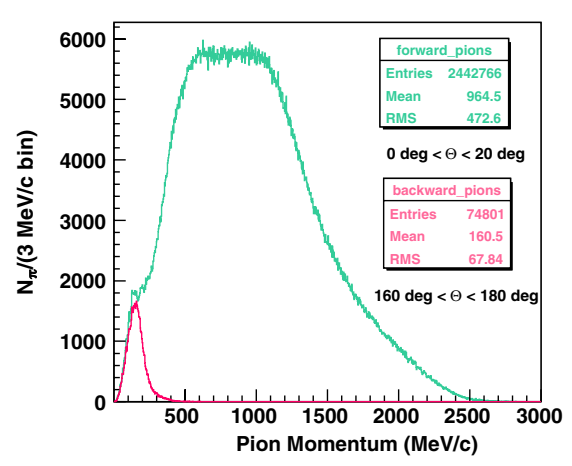

(c) $3 \mathrm{GeV}$ incident protons

FIG. 10. Pion momentum distributions at various incident proton energies.

range $500-800 \mathrm{MeV}$. However, at higher energies it is possible to produce pions in pairs. Double pion production reactions occur only when there is sufficient energy in the collision, and are typical for proton energies beyond $1 \mathrm{GeV}$. Momentum and energy spectra of the pions produced by various energy protons incident on a graphite target show the onset of the double pion production at $750 \mathrm{MeV}$ (Fig. 8). However, the double pion production peak can be seen clearly on pion momentum and energy spectra from $1 \mathrm{GeV}$ proton energy onwards (Fig. 9).

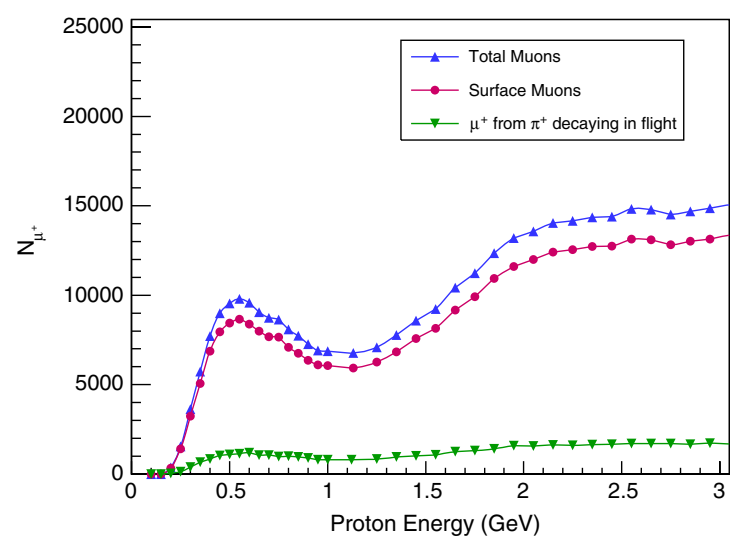

FIG. 11. Variation of muon yield with proton energy.

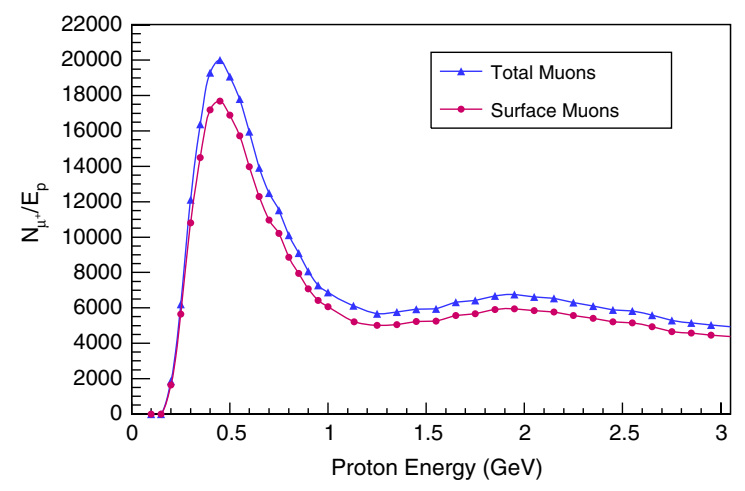

FIG. 12. Normalization to the incident proton energy.
The momentum spectrum and angular distribution of the pions produced depend on the primary proton beam energy; therefore simulations were performed for several incoming proton energies. The pions exiting the target at angles smaller than 20 degrees and higher than 160 degrees with respect to the proton beam were recorded (Fig. 10). These figures show the momentum distributions of the pions produced by incident proton beam energies at TRIUMF, ISIS, and J-PARC accelerators. The pions are forward biased and the forward-backward asymmetry is increasing with the energy of the proton beam. The momentum distribution of the pions exiting the target at angles larger than 160 degrees is a single Gaussian. The average momentum increases from $122 \mathrm{MeV} / c$ for $500 \mathrm{MeV}$ protons to $160 \mathrm{MeV} / c$ for $3 \mathrm{GeV}$ protons. For the pions coming out of the target at angles smaller than 20 degrees, the momentum distribution for $3 \mathrm{GeV}$ protons is a superposition of three Gaussians, one centered at $150 \mathrm{MeV} / c$, one at $\sim 500 \mathrm{MeV} / c$, and one at $\sim 1 \mathrm{GeV} / c$.

\section{Muon production}

The muons produced by pions decaying at rest near the target surface have sufficient energy to escape from inside the target and they are known in the literature as surface muons. Only surface positive muons are produced because

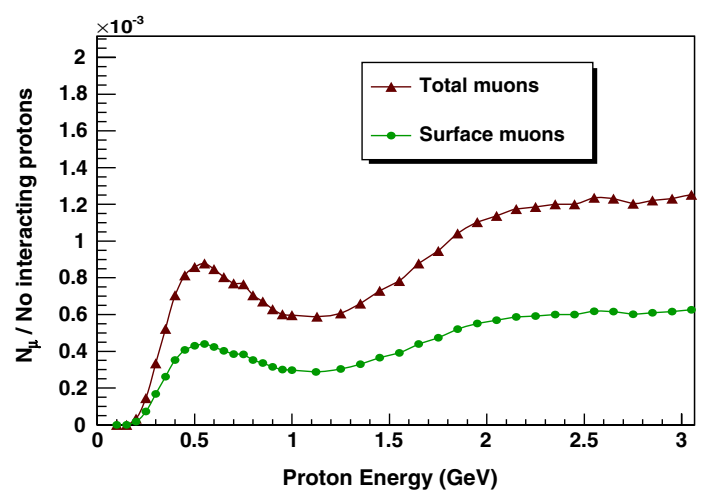

FIG. 13. Normalization to the number of protons interacting in the target. 


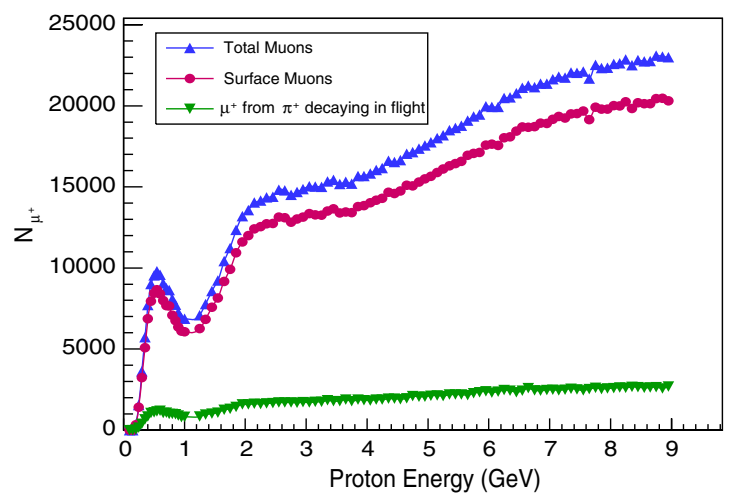

FIG. 14. Variation of muon yield with proton energy at higher energies.

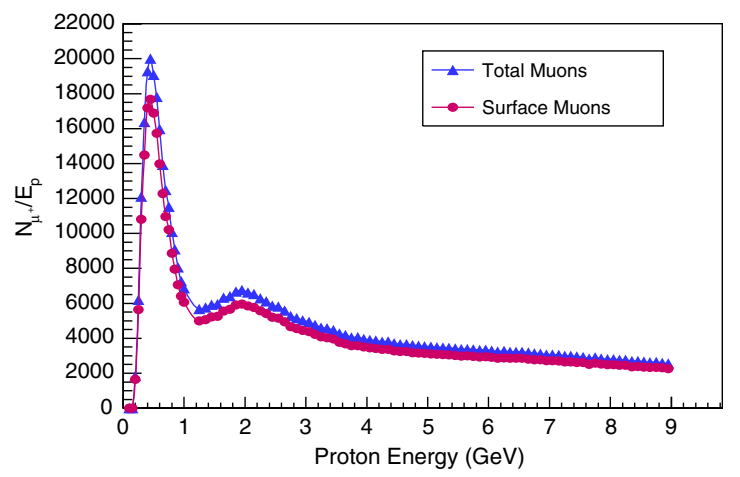

FIG. 15. Normalization of the muon yield to the proton energy.

the negative pions stopped inside the target are captured by the carbon nuclei. The surface muons have a momentum range $0-30 \mathrm{MeV} / c$ and the muon beam has a high intensity due to the high stopping pion density inside the target. In order to detect all the surface muons, the target was surrounded by a spherical shell in the GEANT4 simulations. The shell is made of vacuum to avoid particle scattering and has a minimum radius of $14 \mathrm{~cm}$ and a maximum radius of $16 \mathrm{~cm}$. Figure 11 shows the total muon production rate (surface muons and muons from pions decaying in flight and having a momentum lower than $30 \mathrm{MeV} / c$ ) for various incident proton energies. A peak at about $500 \mathrm{MeV}$ can be observed in the muon production rate.

Increasing the proton energy above this value merely produces more high momentum pions in the forward direction, mostly well outside the momentum range likely to be used by a decay beam, though there is a small increase in the useful range. At higher proton beam energies, most pions have high kinetic energy and escape the target rather than coming to rest and having time to decay to surface muons. A normalization to the incident proton energy is plotted in Fig. 12 and the peak is shown clearly at about $500 \mathrm{MeV}$.

Since the proton transmission is a function of the proton energy, a normalization to the number of protons interacting in the target was done and it also shows a peak at about $500 \mathrm{MeV}$ (Fig. 13). This normalization was done to calculate the average number of muons produced in a proton interaction inside the target for different incident proton energies. Therefore, as the surface muon production is concerned, TRIUMF gets a higher muon production at $500 \mathrm{MeV}$ than ISIS at $800 \mathrm{MeV}$ and J-PARC at $3 \mathrm{GeV}$. Because the muon production rate starts to increase from $1 \mathrm{GeV}$ onwards, the study has been extended to higher proton energies up to $9 \mathrm{GeV}$ in order to look for a second peak in muon production and a continuos increase in muon yield with proton energy was found (Fig. 14). However, the normalization of the muon yield to the incident proton energy shows a single peak at about $500 \mathrm{MeV}$ (Fig. 15); therefore no gain is achieved in going to higher energies for this particular target geometry and material and considering the limitations of proton transmission imposed by the neutron experiments.

The momentum distributions of the surface muons produced by an incident proton beam of energies used at TRIUMF, ISIS, and J-PARC accelerators are shown in Fig. 16. The simulation recorded the surface muons emitted in the forward direction at an angle smaller than 20 degrees with respect to the proton beam and in the

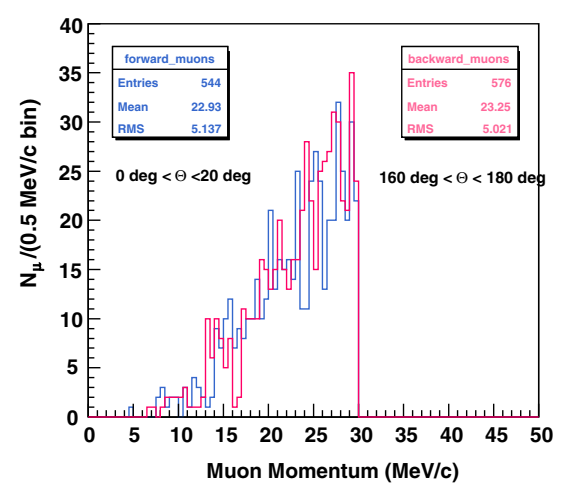

(a) $500 \mathrm{MeV}$ incident protons

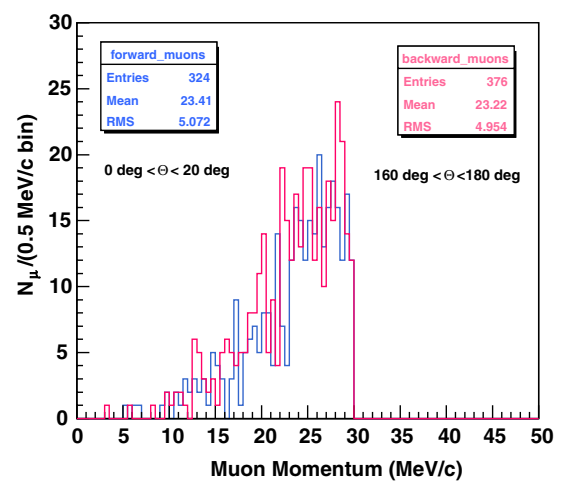

(b) $800 \mathrm{MeV}$ incident protons

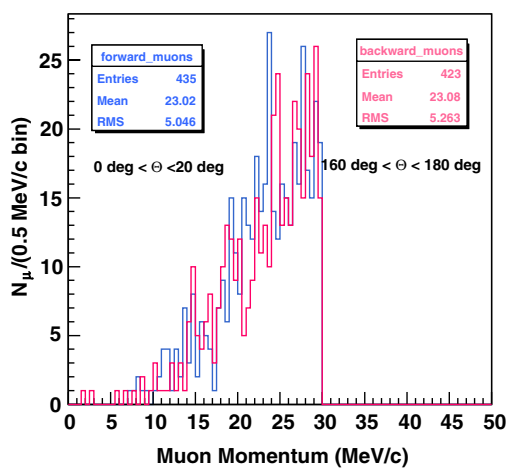

(c) $3 \mathrm{GeV}$ incident protons

FIG. 16. Surface muon momentum distributions for various incident proton energies. 
backward direction at an angle higher than 160 degrees. As far as the pion production is concerned, the forwardbackward asymmetry increases with the proton energy, while in the case of muons, the muon rates and momentum distributions are similar for all three proton energies. This suggests that the surface muon production is isotropic.

\section{CONCLUSION}

Muon production rates as a function of proton beam energy were investigated in this paper using the GEANT4 Monte Carlo code. The aim of this study was to determine the optimal incident proton energy for surface muon production and further work will address other aspects of target design (material choice, target geometries). A validation of three GEANT4 theoretical models applicable in the energy range of interest for ISIS shows general good agreement between simulation and experimental data. Although their predictions are similar, the Binary Cascade Model and the INCL-ABLA model have the main disadvantage of a microscopic precision that is CPU intensive and for this reason the Bertini Cascade model was preferred in all simulations. Positive pions were recorded in these simulations regardless of their momentum, while in the case of positive muons, only those with momentum lower than $30 \mathrm{MeV} / c$ were recorded. The pion production increases with the energy of the incident proton beam and studies of the momentum spectrum and angular distribution show that the pions are forward biased, the forward-backward asymmetry increasing with energy. When there is sufficient energy in the collision, double pion production reactions can be observed, typically for energies beyond $1 \mathrm{GeV}$ (the pion momentum and kinetic energy spectra show the onset of double pion production from $750 \mathrm{MeV}$ proton energy onwards). The momentum distribution of the surface muons show that the muon production is isotropic for all energies. Studies of surface muons rate as a function of proton energy up to $9 \mathrm{GeV}$ show a single peak at about $500 \mathrm{MeV}$ (Fig. 15). Increasing the proton energy above this value merely produces more pions mostly well outside the momentum range likely to be used by a decay beam. Therefore no gain is achieved in going to higher energies for this particular target geometry and material. This suggests that $500 \mathrm{MeV}$ proton energy is the optimal energy and one should aim for this value at a stand alone muon facility.

[1] S. L. Lee, S. H. Kilcoyne, and R. Cywinski, Muon Science (Muons in Physics, Chemistry and Materials) (Institute of Physics Publishing, Bristol, Philadelphia, 1999).

[2] E. Cartlidge, Phys. World 19, 13 (2006).

[3] R. Cywinski et al., Physica (Amsterdam) 404B, 1024 (2009).

[4] "Towards a Next Generation European Muon Source," NMI3/CONFORM Workshop, Cockcroft Institute, Daresbury, 2008.

[5] GEANT4-version 4.9.3.p02, CERN [http://geant4.cern.ch].

[6] A. Ribon et al., IEEE Nuclear Science Symposium Conference Record (IEEE, Orlando, FL, 2009).

[7] A. Ribon et al., Report No. CERN-LCGAPP, 2010.

[8] A. Heikkinen, Helsinki Institute of Physics, Internal Report No. HIP-2009-02.

[9] A. Heikkinen, The Monte Carlo Method: Versatility Unbounded in a Dynamic Computing World (American Nuclear Society, Chattanooga, TN, 2005).

[10] G. Folger and J. P. Wellisch, in Proceedings of Computing in High Energy and Nuclear Physics, Interlaken, Switzerland, 2004, Vol. ID256 [Eur. Phys. J. A 21, 407 (2004)].

[11] A. Heikkinen, P. Kaitaniemi and A. Boudard, J. Phys. Conf. Ser. 119, 032024 (2008).

[12] D. R. F. Cochran, P. N. Dean, P. A. M. Gram, E. A. Knapp, E. R. Martin, D. E. Nagle, R. B. Perkins, W. J. Shlaer, H. A. Thiessen, and E. D. Theriot, Phys. Rev. D 6, 3085 (1972).

[13] Adriana Bungau et al., in Proceedings of the International Particle Accelerator Conference, Kyoto, Japan (ICC, Kyoto, 2010), MOPEA075, p. 247.

[14] J. Apostolakis et al., J. Phys. Conf. Ser. 160, 012073 (2009).

[15] Adriana Bungau et al., in Proceedings of the International Particle Accelerator Conference, Kyoto, Japan (Ref. [13]), MOPEA079, p. 259. 